\title{
Comparaison de methodes pour la mesure des matieres en suspension dans les cours d'eau, en présence de sable
}

\author{
Guillaume DRAMAIS, Benoît CAMENEN, Jérôme LE COZ
}

Irstea, UR HHLY, centre de Lyon-Villeurbanne, 5 Rue de la Doua, CS 20244, F-69625 Villeurbanne Cedex, France

e-mail: guillaume.dramais@irstea.fr

\begin{abstract}
RÉSUMÉ. - La mesure des concentrations de matières en suspension (MES) dans les cours d'eau est un sujet d'étude complexe. Des questions se posent sur la répartition spatiale des particules dans le cours d'eau, notamment celle du sable en suspension. La taille des particules et leurs propriétés, mais aussi la capacité de la rivière à mobiliser ces matières sont des sujets qui intéressent la recherche et les gestionnaires de cours d'eau. Dans cette étude, nous nous focaliserons sur la mesure de la concentration et de la distribution granulométrique des MES non-organiques dans un échantillon prélevé manuellement ou de manière automatique dans un cours d'eau. Plusieurs méthodes de dosage en laboratoire existent dont la norme NF EN 872 préconisée pour la mesure des concentrations en MES dans la plupart des types d'eau. Cependant cette méthode présente des limites importantes en cas de présence de particules de diamètre supérieur à $63 \mu \mathrm{m}$ (sables). Nous illustrons dans cette étude le problème du sous-échantillonnage en présence de sable, en testant différentes méthodes utilisées en France et ailleurs sur une centaine d'échantillons de concentrations variées prélevés dans une rivière alpine. Notre étude montre que la méthode ASTM D3977 est plus précise que les autres méthodes testées pour mesurer les concentrations en MES en présence de sable dans l'échantillon.
\end{abstract}

Mots-clés : matière en suspension (MES), sable, norme NF EN 872, sous-échantillonnage, tamisage

\section{Methods comparison for river suspended sediment measurements containing sand}

\begin{abstract}
Measurements of SSC (Suspended Solid Concentration) concentrations in rivers are a complex scientific issue. Many questions arise on the spatial and temporal distribution of particles throughout a cross-section, on the properties of particles and grain-size, and also on the sediment transport capacity of streams and rivers. In this study, we focused on the SSC and grain size distribution measured from river samples, automatically or manually acquired. Many agencies suggested slightly different methods for measuring SSC: The European standard NF EN 872, which is similar to the US EPA 160.2 requires sub-sampling using shake-and-pour aliquot selection. The APHA 2540D requires sub-sampling by pipetting at mid-depth in the original sample shaken with a magnetic stirrer. These methods lead to significant uncertainty when particles larger than $63 \mu \mathrm{m}$ are present in the samples. The ASTM D3977 analysis method, endorsed by the USGS is more accurate to capture and quantify particles larger than $63 \mu \mathrm{m}$. We confirm in this study the sub-sampling problem in a large concentration range using a set of samples from an alpine river.
\end{abstract}

Key-words: suspended solid concentration (SSC), sand, NF EN 872 standard, sub-sampling, sieving

\section{INTRODUCTION}

\section{I.1. Matières en suspension dans les cours d'eau}

La mesure des matières en suspension (MES) dans les rivières intéresse les gestionnaires de cours d'eau et les scientifiques pour répondre à différentes problématiques comme la gestion des érosions et des dépôts dans les chenaux de navigation, le transfert et les flux de polluants, la gestion d'ouvrages, etc. Les MES désignent l'ensemble des matières solides insolubles présentes dans un échantillon liquide. Elles peuvent être constituées de matières minérales (argiles, limons, sables) mais aussi organiques. Le sable qui constitue une partie des MES, est un matériau minéral, granulaire, issu principalement de l'érosion du bassin versant. Il peut être transporté dans les rivières par charriage, en saltation et en suspension. Selon plusieurs classifications dont celle de Hjulström [1935], la taille des particules de sable se situe entre $0,063 \mathrm{~mm}$ (limon) et $2 \mathrm{~mm}$ (gravier). Sa mobilisation par l'écoulement est généralement intermittente, en fonction de sa disponibilité, des conditions hydrodynamiques, de la géométrie et de la nature du lit de la rivière. De plus, le sable en suspension n'est pas réparti de façon homogène dans la section; on parle de suspension graduée avec généralement une augmentation de la concentration en se rapprochant du lit de la rivière. On différencie ainsi la suspension graduée de la suspension homogène de lessivage (ou « washload» en anglais) constituée de particules plus fines que les sables (argiles, limons), réparties de façon homogène dans la section et mobilisées plus facilement par les écoulements dans les bassins versants. Si la suspension graduée de sable est en équilibre direct avec l'hydrodynamique locale, la suspension de lessivage dépend moins de la capacité de transport locale de l'écoulement que des apports amont. Philippe Lefort [1991] décrivait le sable comme un " passager clandestin » car il est encore difficile de le mesurer et de modéliser les processus liés à ses déplacements dans le cours d'eau.

\section{I.2. Prélèvement des échantillons}

Afin de pouvoir mesurer la concentration et la granulométrie des MES, des échantillons sont prélevés, par 
différents moyens : préleveurs automatiques, prélèvements manuels en berge ou depuis des ponts, préleveurs isocinétiques (Figure 1). La forme de ces derniers permet de ne pas modifier les vitesses d'approche de l'eau autour de l'entrée du préleveur, afin de ne pas biaiser l'échantillon. Les ingénieurs et scientifiques ont conçu des préleveurs d'eau et de sédiments depuis le début du $\mathrm{XX}^{\text {ème }}$ siècle. L'USGS (United State Geological Survey), dans ce domaine a ainsi mis au point et documenté de nombreux préleveurs [Gray et Landers, 2014], intégratifs ou ponctuels (Figure 1c), à fermeture activée à distance ou pas. Certaines équipes utilisent des préleveurs qui vont piéger instantanément un volume d'eau comme les bouteilles type Van Dorn, Niskin (Figure 1b) ou Callède (Figure 1a). D'autres préleveurs vont permettre d'échantillonner un volume pendant un temps d'exposition variable (fonction des vitesses d'approche et du volume cible) comme ceux de l'OFEV (Figure 1e, Office Fédéral de l'Environnement, $\mathrm{CH}$ ) qui permet d'échantillonner 1L, ou la bouteille de Delft (Figure 1d) qui a été mise au point par l'université de Delft [Dijkman, 1978]. C'est un décanteur intégratif isocinétique qui permet de prélever et de déterminer un flux de sable en fonction d'un temps d'exposition et de la vitesse d'écoulement à l'approche du dispositif. Certaines équipes effectuent des prélèvements par pompage, depuis des embarcations avec des crépines lestées ou comme sur la Figure 1f à l'aide de dispositif de maintien de la crépine dans l'écoulement. Les systèmes de pompage et préleveurs automatiques permettent une automatisation des prélèvements avec des possibilités d'asservissement à d'autres capteurs ou instruments. Le choix du type de prélèvement est souvent dicté par les conditions hydrologiques, les moyens de déploiement disponibles (humain et matériel) et l'objectif de l'étude.

\section{I.3. Sous-échantillonnage et analyse des échantillons}

Une fois l'échantillon prélevé, un sous-échantillonnage est souvent nécessaire pour analyser séparément les différents paramètres (concentration, granulométrie, etc.). Le sous-échantillonnage consiste, à partir d'un échantillon d'eau prélevé en rivière, à le fractionner pour effectuer des analyses. Plusieurs méthodes existent pour ce faire, la principale difficulté étant de créer des sous-échantillons homogènes et représentatifs de l'échantillon mère (cf. Section II. Méthodes). Des appareils séparateurs d'échantillons (cone splitter, churn splitter) existent et permettent de diviser l'échantillon de manière gravitaire. Ils n'ont pas été testés dans cette étude mais l'ont été dans des laboratoires américains [Capel, 1996 ; Horowitz, 1997].

L'analyse la plus courante réalisée sur ces sous-échantillons est la mesure de la concentration en MES. Elle peut être réalisée, là encore, par différentes méthodes, la méthode la plus courante étant la filtration du sous-échantillon sur filtre en fibre de verre. Cette technique permet d'isoler puis peser la partie solide de l'échantillon; on obtient ainsi une (a)

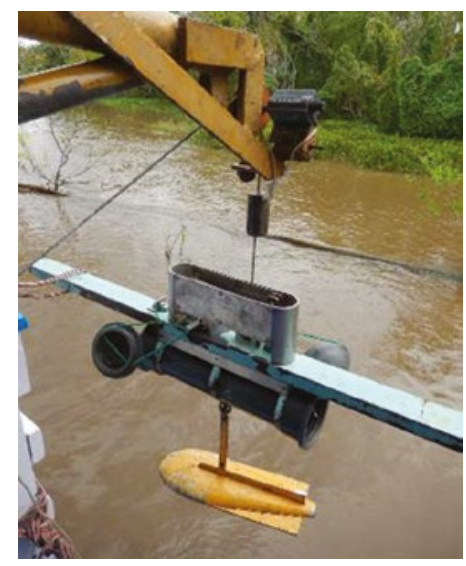

(d)

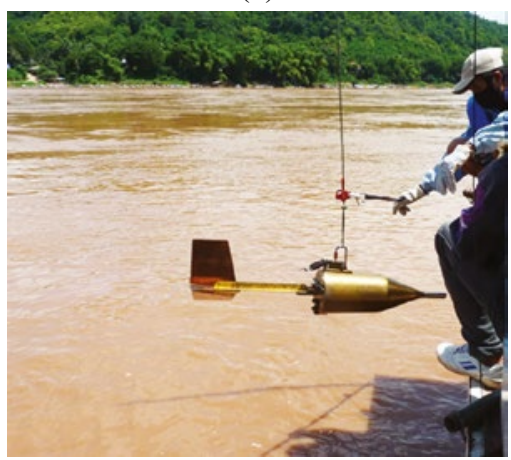

(b)

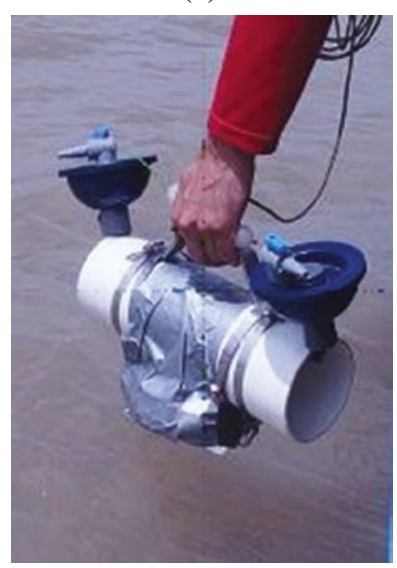

(e)

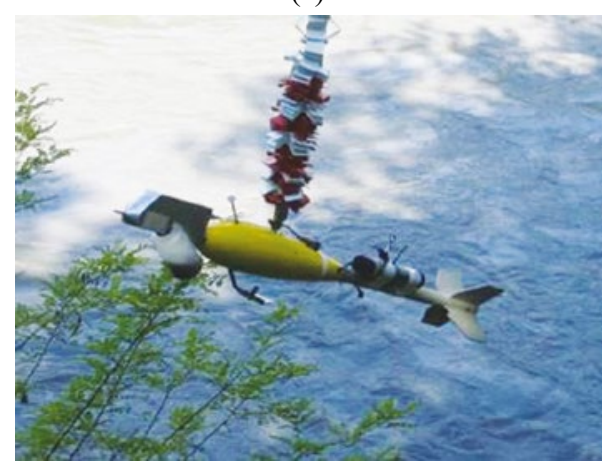

(c)

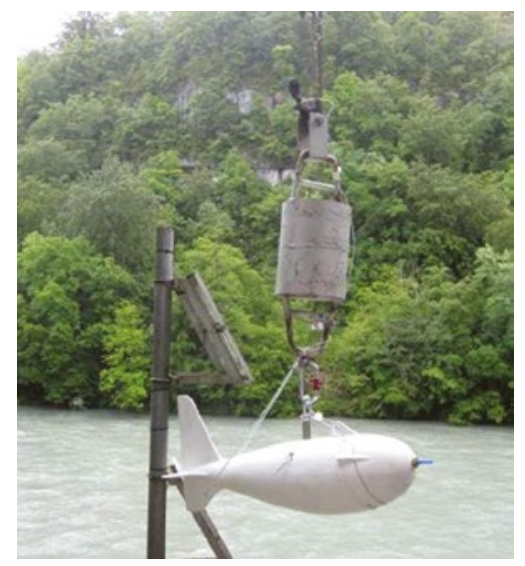

(f)

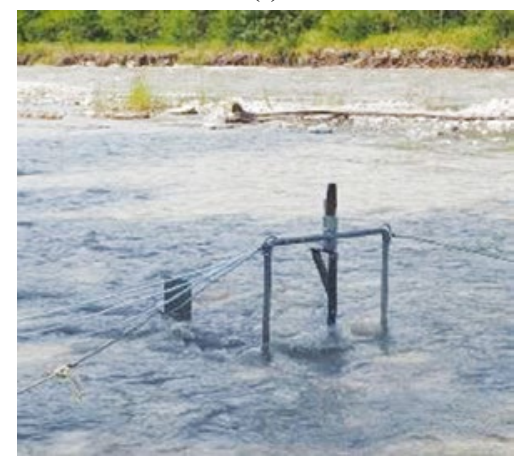

Figure 1 : Différents types de préleveurs déployés par les auteurs et en collaboration avec la CNR, l'équipe de l'observatoire HyBAM, l'IRD, le Cerege, l'OFEV: (a) Préleveur type Callède (capacité 8L) déployé sur l'Amazone en 2014, (b) Préleveur manuel type Niskin (2L) déployé sur la Sä̈gon River en 2016, (c) P72 (0,5L) déployé depuis la traille de Bognes sur le Rhône en 2016, (d) Préleveur de sable intégratif type Delft déployé sur le Mékong en 2013, (e) Préleveur automatique de l'OFEV (1L) déployé aux Rippes sur le Rhône en 2016, (f) Préleveur multiprofondeur par pompage déployé sur l'Arc en Maurienne en 2017. 
concentration massique en MES. Pour vérifier la cohérence du sous-échantillonnage et la sensibilité des protocoles d'analyses, les laboratoires effectuent des réplicas, des triplicas, et certains testent l'influence du volume d'échantillon sur la répétabilité du résultat de MES [Versini, 2015].

C'est la technique de séparation des MES dans un sous-échantillon qui sera étudiée dans cet article. La séparation des MES peut se faire par filtration, par centrifugation ou encore par évaporation (voir Section II). Le paragraphe suivant passe en revue des techniques de mesure de la concentration en MES alternatives à la technique de séparation des MES, qui reste la plus couramment employée en pratique.

\section{I.4. Revue des méthodes alternatives pour la mesure des concentrations en MES}

D'autres méthodes de mesure permettent la mesure indirecte de la concentration en MES en utilisant la densité ou la masse volumique du fluide comparées à celles de l'eau pure sous conditions de température et de pression identiques :

L'hydromètre mesure de façon directe la densité du fluide, il consiste en un cylindre creux, lesté et gradué, qui s'enfonce plus ou moins dans le liquide à mesurer selon sa densité, par le jeu de la poussée d'Archimède. On lit directement la densité du liquide sur la graduation de l'hydromètre présente à la surface libre du bécher ou $\mathrm{du}$ flacon. L'hydromètre est une des méthodes préconisée par la norme ISO 4365 [ISO, 2005], référencée dans le Tableau 1.
Une autre mesure directe de la densité du fluide est possible via une mesure de pression différentielle entre deux points distants verticalement dans la colonne d'eau. Cette méthode est utilisée pour mesurer en continu la concentration dans les cours d'eau chargés en MES [Gray, 2010]. En pratique, elle pose encore des problèmes de fiabilité et de sensibilité dans les cours d'eau.

Le pycnomètre est une méthode de mesure différentielle de masse d'un volume connu d'eau et de sédiments. Cette méthode validée par Niel [1985] par comparaison avec l'ancienne version de la norme NF EN 872 [2005] pour des concentrations supérieures à $0,3 \mathrm{~g} / 1$ est encore utilisée pour des suivis en temps réel de concentrations fortes, pendant des chasses de barrage notamment. Cette méthode peut être utilisée dans un laboratoire improvisé sur le terrain.

Il existe plusieurs types de densimètres. Les densimètres à force de Coriolis, permettent de calculer une masse volumique du fluide qui passe dans une conduite, grâce à des mesures de vibration sur le fluide en mouvement. Le principe de mesure des densimètres à fréquence d'oscillation est, quant à lui basé sur le maintien en oscillation d'un tube en $U$ dont la mesure de la fréquence résultante est directement proportionnelle à la masse volumique du fluide injecté. D'autres densimètres utilisent des méthodes de radiométrie par rayon gamma qui sont atténués en traversant le fluide. L'atténuation est liée à la densité du fluide. Les densimètres sont plutôt utilisés en conduites fermées dans les processus industriels [Lardière, 2009] et sont encore difficilement adaptables à des contextes de mesure en milieu naturel.

Tableau 1 : Représentation schématique des protocoles de mesure normalisés des concentrations massiques en MES. Les méthodes alternatives proposées dans la norme ISO 11657 [ISO, 2014] ne sont pas représentées.

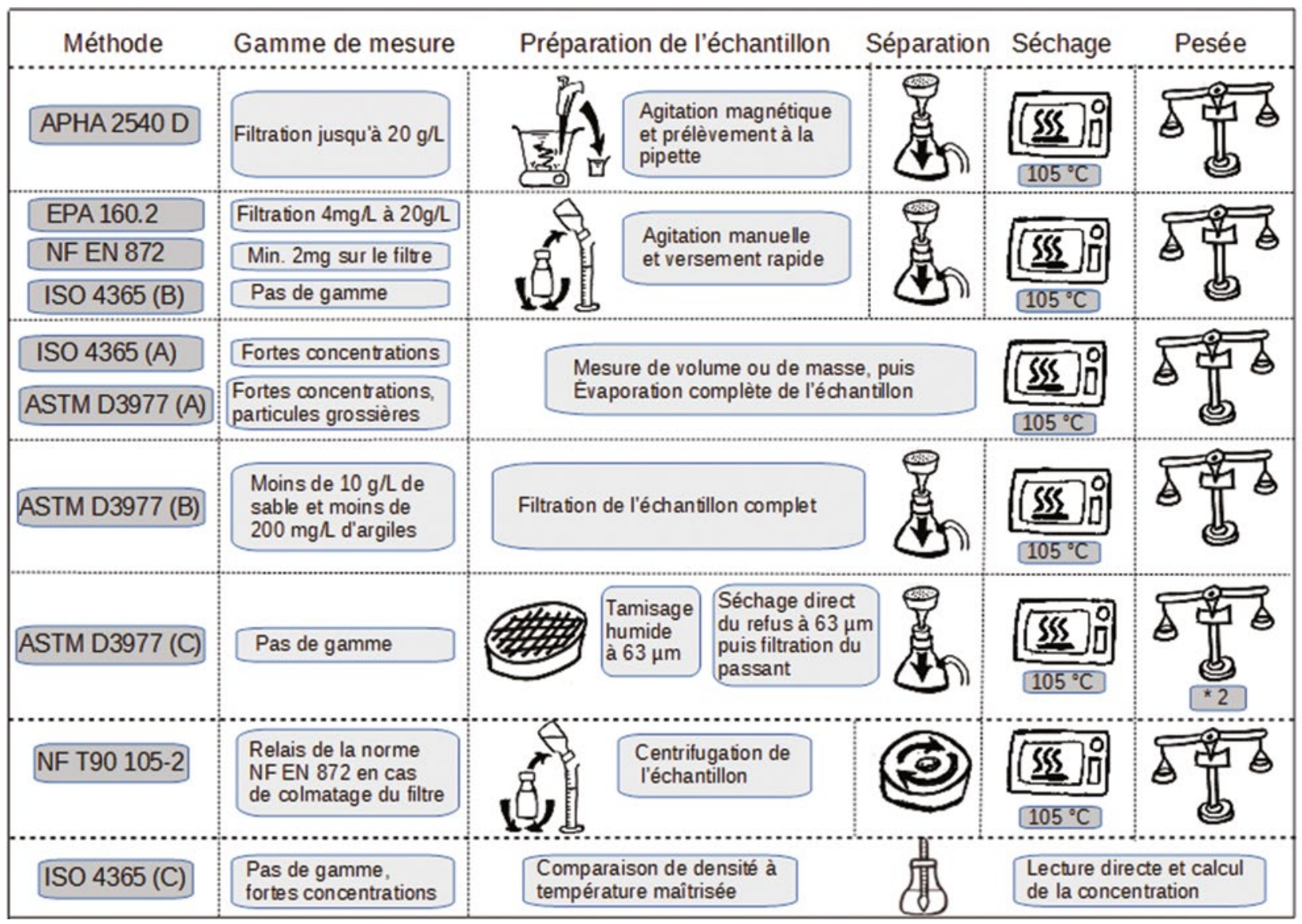


D'autres instruments utilisent les propriétés optiques du fluide pour déterminer une turbidité. Ainsi, le disque de Secchi mis au point au XIX ${ }^{\text {ème }}$ siècle sert encore à évaluer la profondeur de pénétration verticale de la lumière dans l'eau. La mesure consiste à déterminer la profondeur à laquelle un disque blanc et noir devient invisible à l'œil nu. Cette méthode est peu précise mais très simple à utiliser, souvent depuis une embarcation.

Les turbidimètres et transmissiomètres [ISO, 2016] utilisent les propriétés optiques d'absorption et de diffusion de la lumière par les particules présentes dans l'eau. La sensibilité de ces différentes mesures dépend fortement de la taille et de la nature des particules présentes dans la colonne d'eau et les correspondances entre elles ne sont pas univoques. Ces instruments permettent cependant une fréquence d'acquisition très importante et sont aujourd'hui couramment utilisés dans les stations de mesure en rivière, associés à des préleveurs automatiques pour l'élaboration de courbes de conversion entre concentration en MES et turbidité [Thollet, 2013].

D'autres instruments pour la mesure in-situ des concentrations en MES sont développés actuellement avec des capteurs acoustiques ADCP (Acoustic Doppler Current Profiler), ABS (Acoustic Backscatter System) ou utilisant l'atténuation laser. Ils permettent d'évaluer la répartition et la concentration en MES surtout pour les particules les plus grosses. Ces méthodes nécessitent encore des phases de calibration coûteuses en temps pour des résultats encore imprécis en rivière [Gray, 2014]. Certaines de ces méthodes alternatives sont décrites dans la norme ISO 11657 [ISO, 2014], parfois comparées entre elles, partiellement validées par telle ou telle campagne de mesure mais aucune ne s'impose actuellement comme référence.

Toutes ces techniques alternatives sont utilisées ou développées pour des applications variées ; de recherches ; opérationnelles ou de diagnostic. Elles sont choisies en fonction de critères qui peuvent être les objectifs de l'étude, l'environnement de mesure, les capacités de déploiement, le coût des instruments.

\section{I.5. Objectifs de l'étude}

Les données de concentration en MES, une fois acquises, sont exploitées pour établir des relations de conversion entre MES et mesures de turbidité, calculer des bilans de flux de MES, de polluants associés, ou pour mieux comprendre la dynamique sédimentaire. Souvent les scientifiques s'intéressent à la concentration en MES mais aussi à la taille et la composition des particules dont les propriétés vont varier (adsorption des polluants, floculation, présence de matière organique, de sable). Il existe cependant des incertitudes non-négligeables sur l'estimation de la concentration et de la granulométrie des MES selon la méthodologie utilisée, en particulier du fait du sous-échantillonnage. Nous proposons dans cette étude une comparaison et discussion des méthodes de mesures des concentrations en MES utilisées en France et des conséquences sur l'analyse des particules en suspension les plus grossières.

Nous détaillerons dans un premier temps les limites de la norme NF EN 872 [2005], référence en vigueur en France et en Europe. Nous évaluerons les erreurs et les conséquences liées au sous-échantillonnage et essaierons de trouver des moyens de les réduire. La présence de sable (particules $>63 \mu \mathrm{m}$ ) dans les échantillons va considérablement influencer les performances des différentes méthodes ; nous essayerons de quantifier dans quelle mesure.

\section{MÉTHODES DE MESURE DE LA CONCENTRATION EN MES}

\section{II.1. Généralités}

La mesure directe de la concentration massique en MES dans un échantillon liquide est décrite par plusieurs normes (Tableau 1) que nous allons détailler. Plusieurs points sont communs ou singuliers aux différentes méthodes :

- Ces méthodes diffèrent surtout dans la préparation des échantillons analysés. Deux choix sont possibles : utiliser la totalité de l'échantillon prélevé ou considérer un sous échantillon aussi appelé aliquote comme représentatif.

- Après la sélection de l'échantillon, il convient de déterminer un volume à analyser. Toutes ces méthodes (excepté celle de l'hydromètre, cf. I.4) sont basées sur la séparation des phases solides et liquides de l'échantillon : par filtration, évaporation ou centrifugation.

- Après séparation on retrouve dans chaque protocole des phases de séchage et de pesée qui vont permettre de déterminer la masse sèche de sédiments présente dans le volume d'analyse.

Le Tableau 1 récapitule les différentes normes, leurs gammes de validité théoriques, et quelques étapes clefs des protocoles. Ne sont pas citées dans ce tableau, les étapes importantes de conservation des échantillons, de qualité et de préparation des filtres avant analyse, de refroidissement au dessiccateur, qui sont communes aux différentes approches.

\section{II.2. La préparation par sous-échantillonnage}

La plus courante des méthodes de sous-échantillonnage consiste à " Agiter vigoureusement le flacon et transférer immédiatement et d'un seul trait un volume convenable d'échantillon dans une éprouvette graduée ", ce protocole est commun aux normes NF EN 872 [AFNOR, 2005], EPA 160.2. [USEPA, 1999] et ISO 4365 (B) [2005]. Une autre méthode utilisée classiquement consiste à prélever à la pipette un sous-échantillon dans un bécher muni d'un agitateur magnétique, en prélevant à mi-profondeur du mélange entre le vortex créé par l'agitation et la paroi du bécher [APHA, 2005]. Une autre méthode aussi testée dans cette étude consiste à verser l'échantillon dans un seau et ensuite à l'aide d'une louche, ou d'un flacon sans col, d'effectuer des mouvements aléatoires en forme de « 8 » dans les trois dimensions dans le seau et de retirer un sous échantillon, placé ensuite dans une éprouvette graduée. Cette méthode de sous-échantillonnage est inspirée des pratiques de l'équipe IRD de l'observatoire Hybam [Armijos, 2017]. Nous testerons et identifierons cette méthode de sous-échantillonnage par la suite comme : " Agitation-prélèvement 》. Il est à noter que l'équipe Hybam utilise cette méthode de sous-échantillonnage, après tamisage à $63 \mu \mathrm{m}$, sur le mélange eau/particules fines pour ne pas avoir à filtrer la totalité du prélèvement. Ce protocole diffère des essais menés dans cette étude, où le sous-échantillonnage est réalisé sur l'échantillon brut.

\section{II.3. La séparation des phases solides et liquides}

\section{II.3.1. La filtration}

La norme française et européenne NF EN 872 [AFNOR, 2005] décrit la procédure de filtration sur filtre en fibre de verre d'un sous-échantillon adapté pour la détermination de la concentration en matières en suspension. Les normes 
américaines APHA SM 2540D [APHA, 2005] et EPA 160.2 [USEPA, 1999] décrivent également une méthodologie de filtration de ce type. Une norme internationale existe, la norme ISO 4365 [ISO, 2005] ; elle propose plusieurs options de séparation des phases liquides et solides en fonction de la concentration des échantillons, mais sans préciser de gamme de validité. L'option $B$ décrit la filtration avec une variante sur filtre papier et l'autre sur filtre en fibre de verre. Aux Etats-Unis-d'Amérique, la norme ASTM D3977 [ASTM, 2007] propose trois variantes de séparation des phases dont deux par filtration : la première (B) par filtration totale de l'échantillon et la seconde (C) qui consiste à effectuer un tamisage à $63 \mu \mathrm{m}$ puis une filtration des fines. C'est cette dernière option que nous considérerons comme référence dans cette étude car elle permet de séparer la totalité des sables du reste des sédiments plus fins sans aucune perte.

\section{II.3.2. L'évaporation}

L'évaporation totale de l'eau présente dans l'échantillon est une solution intéressante pour déterminer la concentration massique en MES dans l'échantillon. Cette méthode est proposée par les normes ISO 4365 [ISO, 2005], option A et ASTM D3977-97 [ASTM, 2007], option A également. Cette méthode n'a pas été testée dans cette étude car nous nous sommes focalisé sur le problème du sous-échantillonnage. Les méthodes par évaporation totale permettent de mesurer la SSC (II.4), en prenant en compte les matières dissoutes ce qui peut avoir une influence sur les analyses de concentrations très faibles.

\section{II.3.3. La centrifugation}

La centrifugation permet également de séparer les matières solides de la phase liquide. Elle est décrite dans la norme française NF T90-105-2 [AFNOR, 1997] et est utilisée pour les eaux chargées pour prendre le relai de la norme NF EN 872 [AFNOR, 2005] en cas de colmatage des filtres (temps de filtration supérieur à $30 \mathrm{~min}$ ).

\section{II.4. Travaux antérieurs sur l'analyse des MES}

Dans les définitions de la norme FD ISO 6107-2 [ISO, 2006], les MES sont définies comme «matières éliminées par filtration ou centrifugation dans des conditions définies ». Les matières solides totales quant à elles, sont définies comme la somme des matières dissoutes et des matières en suspension. Aux Etats-Unis-d'Amérique, la norme ASTM D3977 [ASTM, 2007] définit la concentration en MES (Suspended Solid Concentration, SSC) comme la masse sèche des matières solides et dissoutes présentes dans un échantillon analysé. La norme APHA SM 2540D [APHA, 2005] décrit la procédure de mesure des matières solides totales (Total Suspended Solids, TSS) qui sont définies comme les matières solides retenues par un filtre standard. Plusieurs études ont tenté de mettre en évidence des écarts entre les résultats obtenus par les différents protocoles proposés par les normes de type «TSS et SSC ». Ainsi les travaux de Gray et al. [2000] effectués sur 3200 paires d'échantillons prélevés dans les rivières des États-Unis ont permis de comparer les méthodes de détermination de la SSC et des TSS. Ils ont montré que les méthodes de mesure de SSC étaient plus fiables que les méthodes de mesure type TSS en particulier en présence de sable. Ils ont remarqué une sous-évaluation de la concentration sur les analyses ayant subi un sous-échantillonnage par versement rapide ou par pipette (de 25 à $34 \%$ ). Cette étude déconseille fortement l'utilisation des méthodes TSS pour mesurer la concentration en MES dans les eaux « naturelles » par opposition aux eaux issues des réseaux d'eaux usées. Ces résultats sont confirmés par Glysson et al. [2002] sur une comparaison de 14466 paires d'échantillons. Ils indiquent les erreurs considérables qui peuvent découler des analyses par les méthodes TSS sur les bilans sédimentaires. Guo [2007] a réalisé une étude comparative avec des échantillons artificiels représentatifs des concentrations et tailles de particules que l'on retrouve dans l'environnement. Il s'intéresse à trois méthodes (ASTM [2007], APHA [2005] et USEPA [1999]) et met en évidence des corrélations acceptables entre les mesures de SSC préconisées par la norme ASTM [2007] et les mesures de TSS de la norme USEPA [1999] mais décèle des erreurs importantes sur la méthode APHA [2005], liées au sous-échantillonnage par pipettage. Cette erreur augmente avec la taille des particules. Cette conclusion est confirmée par Clark et al. [2007] qui s'interrogent également sur la position de la pipette lors du sous-échantillonnage [Clark, 2008]. Une comparaison de 215 paires d'échantillons est réalisée en testant l'influence de la position lors du sous-échantillonnage pour évaluer les biais. Clark [2008] tempère les conclusions des études de Gray [2000] et Glysson [2002] en admettant que les méthodes TSS sont bien adaptées aux mesures des concentrations en MES en suspension homogène et les méthodes SSC aux mesures d'échantillons dont on veut connaître de façon plus exacte la concentration totale en MES. Selon elle, la méthode doit être adaptée aux objectifs de l'étude. Selbig et al. [2011] ont contribué à ce débat en étudiant l'influence de la granulométrie et l'établissement de facteurs de correction pour passer de SSC à TSS et ont confirmé les limites des méthodes TSS pour mesurer les concentrations en présence de particules grossières dans l'échantillon. Ces études montrent la sensibilité de ces méthodes d'analyse en particulier pour les échantillons de rivière chargés, notamment avec des particules en suspension de taille importante (sables). En France certains laboratoires utilisent les normes américaines [APHA, 2005] pour des besoins de sous-échantillonnage, ou des variantes de la méthode ASTM [2007], pour éviter justement le sous-échantillonnage. Certaines équipes pratiquent des variantes simplifiées (non normalisées) comme celle dite " de la crêpe » qui est une méthode alternative de filtration, (après sous-échantillonnage par versement rapide) puis séchage rapide du filtre sur plaque chauffante ou au four micro-onde, qui permet d'obtenir une concentration en MES rapidement dans un laboratoire improvisé sur le terrain par exemple, cette méthode a été mise au point et validée lors d'une étude [Cemagref, 1981].

\section{MATÉRIEL ET MÉTHODES}

\section{III.1. Site d'étude}

L'Arc en Maurienne (surface du bassin versant: $1985 \mathrm{~km}^{2}$ à Chamousset) est un affluent rive gauche de l'Isère au régime nival (Figure 2). L'Arc est une rivière très aménagée avec des digues construites notamment pour permettre sa cohabitation avec des infrastructures de transport lourdes (route, autoroute, ligne ferroviaire). L'Arc compte trois barrages au fil de l'eau qui sont chassés chaque année en juin, en période de hautes eaux, afin d'évacuer les sédiments stockés. Le $1^{\text {er }}$ juin 2017, une chasse a été effectuée par l'exploitant des barrages (EDF). Cet évènement contrôlé a été l'occasion de prélever une centaine d'échantillons aux 


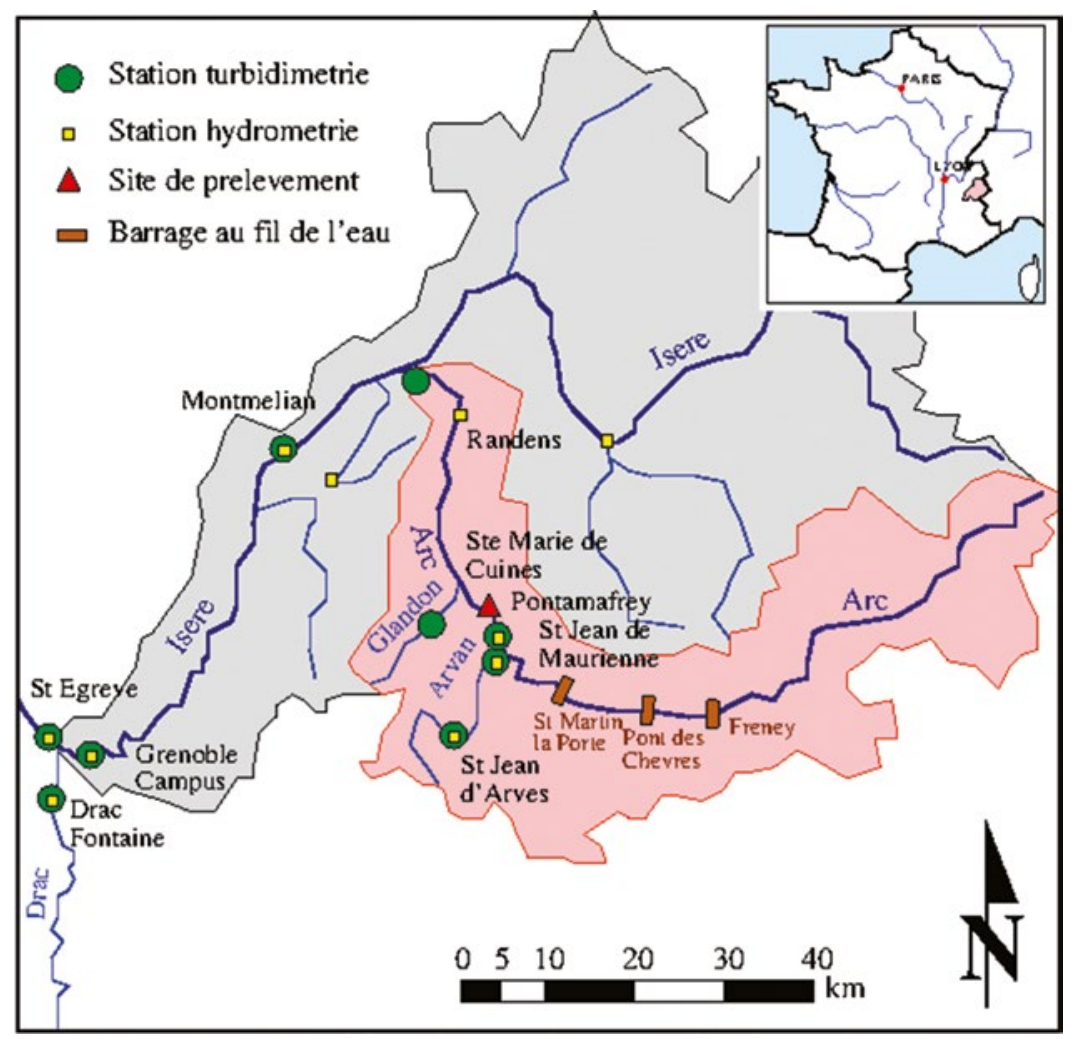

Figure 2 : Localisation du site de prélèvement sur l'Arc-en-Maurienne.

concentrations variées. D'autres échantillons ont été prélevés la veille et les jours suivants pour compléter le jeu de données. Le site expérimental se situe sur la commune de Sainte-Marie-de-Cuines sur un tronçon rectiligne où s'est formé un système de bancs alternés de galets [Jaballah et al., 2015]. Plusieurs études sur la dynamique des sédiments fins ont été réalisées sur ce site [Camenen et al., 2015, 2016]. La lithologie des matériaux présents dans la rivière est complexe car l'Arc traverse une succession de massifs cristallins et des bandes de roches assez diverses (schistes argileux, calcaires, gypses, marnes noires et grès) dominé par des calcaires argileux et des schistes argilo-calcaires en rive gauche [Gidon, 2017]. Dans cette étude, les échantillons analysés ont été prélevés dans la colonne d'eau à l'aide d'un dispositif permettant le pompage simultané de trois échantillons à 5,10 et $20 \mathrm{~cm}$ du fond dans un chenal secondaire (Figure 1f) à partir de préleveurs automatiques. Les autres échantillons sont des échantillons de surface issus de prélèvements manuels en berge et au seau dans le centre de l'écoulement depuis un pont.

\section{III.2. Jeu de données étudié}

Le jeu de données d'une centaine de paires d'échantillons a été analysé au laboratoire HHlab d'Irstea Villeurbanne en concentration en MES et granulométrie laser. Les échantillons secs comportent en moyenne $2,2 \%$ de matière organique, détruite par exposition des échantillons secs dans un four à moufle à $500^{\circ} \mathrm{C}$ pendant 30 minutes (réalisé sur 35 véchantillons). Les échantillons comportent des taux de particules $>63 \mu \mathrm{m}$ variables, de 11 à $90 \%$ de la masse sèche (Figure 3). Dans le cas particulier d'une chasse de l'Arc, il apparaît d'ailleurs qu'une corrélation existe entre les concentrations en MES et le pourcentage de sable dans l'échantillon. Ceci peut s'expliquer du fait que les sables sont mis en suspension pour les contraintes les plus élevées, et donc les débits les plus élevés; de la même manière, les concentrations en MES lors d'un événement de chasse sont fortement corrélées au débit. Il y a plus d'érosion dans les retenues pour les débits les plus élevés, et suivant les conditions d'abaissement du niveau.

On ne différencie pas dans cette étude les sables des flocs éventuels générés par l'agglomération de particules cohésives. Cependant, les photos effectuées au microscope électronique (Figure 4) confirment la présence de sable en majorité dans les matériaux retenus dans le tamis $(63 \mu \mathrm{m})$.

\section{RÉSULTATS : INFLUENCE DES TECHNIQUES DE SOUS-ÉCHANTILLONNAGE}

La comparaison de 28 paires d'échantillons mesurées par les méthodes ASTM (C) avec séparation du sable par tamisage avant filtration et NF EN 872 avec sous-échantillonnage par versement rapide pour les échantillons de l'Arc est conforme aux observations de la littérature, avec une sous-estimation forte de la méthode NF EN 872, (Figure 5a). La Figure $5 \mathrm{~b}$ met en évidence une des raisons de cette sous-estimation : il existe une corrélation forte entre le pourcentage de sable dans la masse totale de sédiments et la différence observée entre les résultats des deux méthodes, en particulier lorsque le pourcentage de sable est supérieur à $50 \%$. Si on considère la méthode ASTM comme référence, la méthode NF EN 872 sous-estime la concentration totale pour les teneurs en sable importantes. On fait des erreurs allant de $25 \%$ à plus de $50 \%$ pour des échantillons contenant plus de $40 \%$ de sable. 


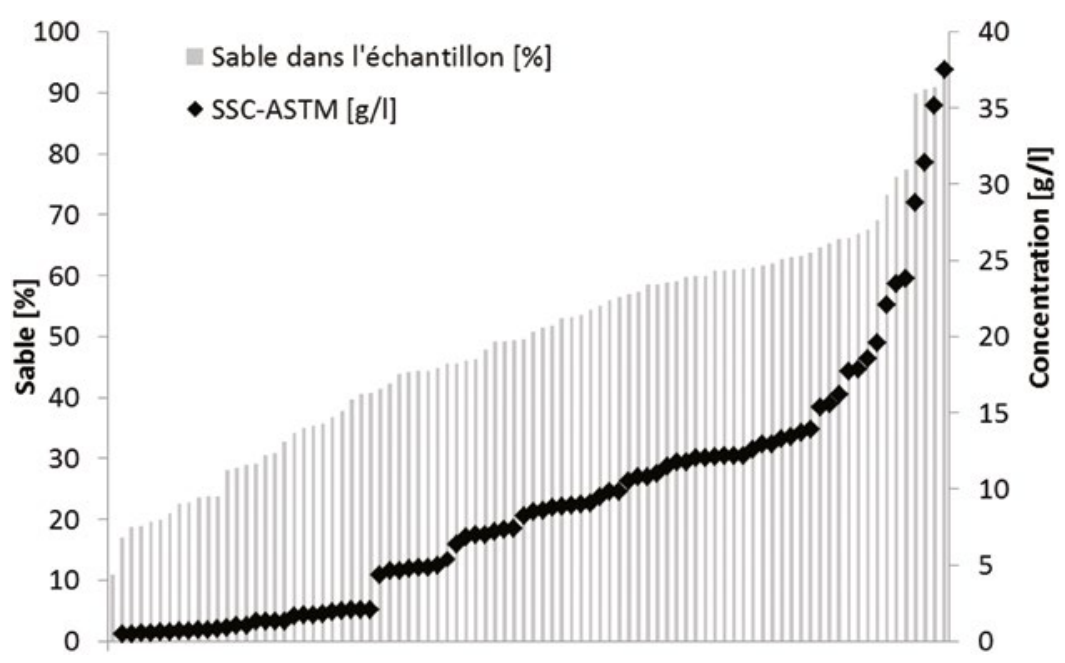

Figure 3 : Proportion de sable (> $63 \mu \mathrm{m}$ ) dans les échantillons et concentration en MES totales [ASTM] du jeu de données complet (classés par concentration croissante).

(a)

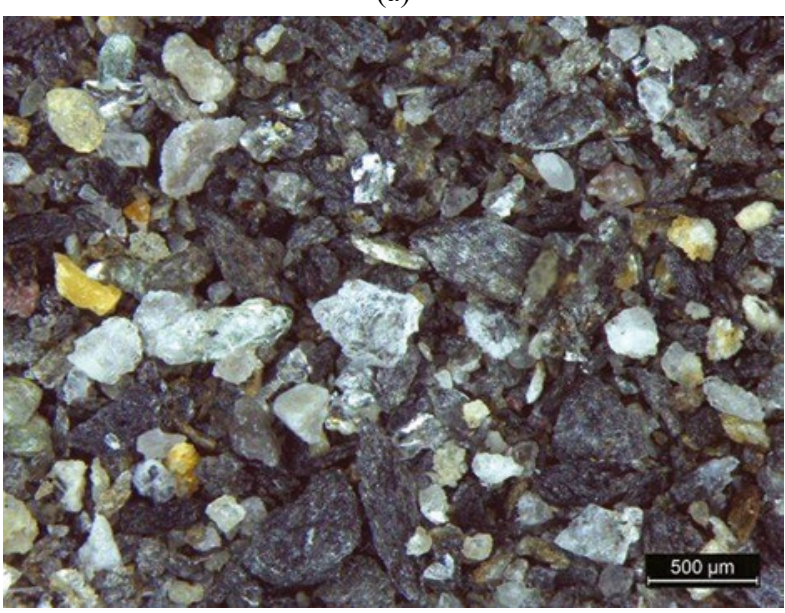

(b)

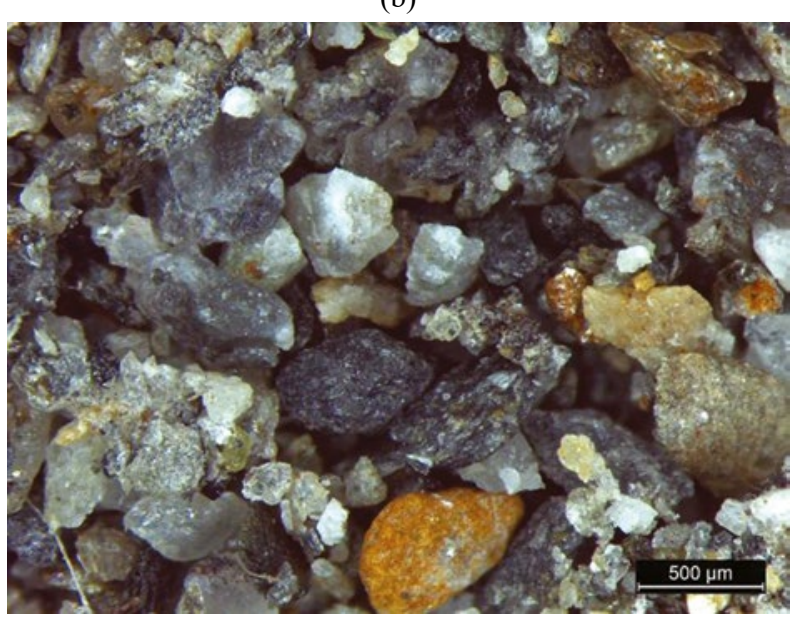

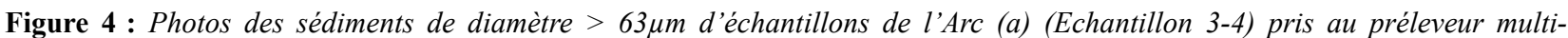
profondeur, (b) (Echantillon Pontamafrey 15h) pris au préleveur automatique en berge.

(a)

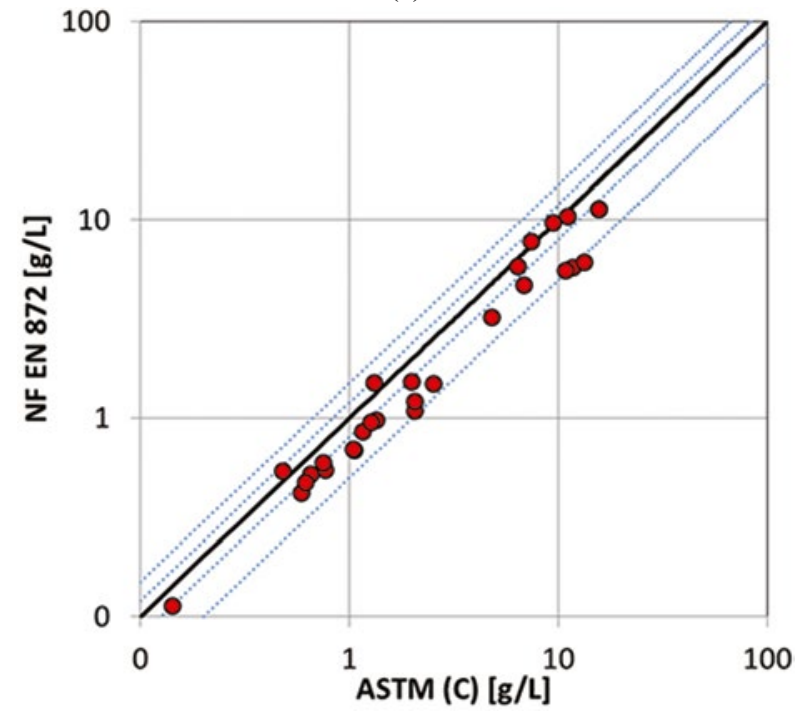

(b)

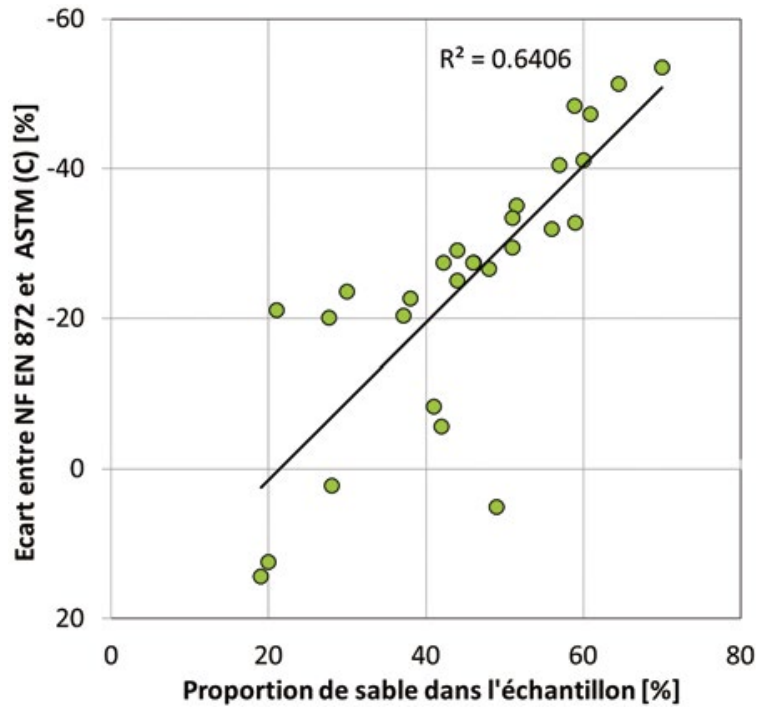

Figure 5 : (a) Comparaison des concentrations ASTM (C) [2007] et NF EN 872 [AFNOR, 2005]. Les traits pointillés bleus sont les enveloppes à \pm 20 et $\pm 50 \%$, (b) écarts calculés en pourcentage entre les concentrations des méthodes NF EN 872 [AFNOR, 2005] et ASTM [2007] en fonction du pourcentage de sable. 
Dans cette comparaison le sous-échantillonnage par agitation et versement rapide est clairement mis en cause dans la sous-estimation des concentrations par la méthode NF EN 872, même pour des échantillons assez peu concentrés (concentration inférieure à $1 \mathrm{~g} / \mathrm{l}$ ). En effet, malgré une agitation vigoureuse les particules les plus lourdes ne restent pas en suspension pendant le temps de versement du sous-échantillon et se déposent au fond du flacon, entraînant une sous-estimation de ces dernières dans l'échantillon analysé. Les concentrations de quatre échantillons sont néanmoins surestimées par la méthode NF EN 872. Il s'agit, pour trois d'entre eux, des échantillons les moins concentrés. Il est possible que dans les échantillons les moins concentrés en MES (et en sable également) le faible nombre de grains dans le sous-échantillon rende leur capture aléatoire et puisse biaiser la mesure dans le sens d'une surestimation comme d'une sous-estimation. Deux autres méthodes de sous-échantillonnage sont ici testées sur 35 paires d'échantillons afin d'en appréhender l'efficacité sur des jeux d'échantillons de concentration variées. La méthode par agitation magnétique et pipettage [APHA, 2005] décrite dans la partie II.2, ainsi que la méthode dite " Agitation-prélèvement », décrite dans la partie II.2.

Les résultats des deux méthodes de sous-échantillonnage testées sont présentés sur les Figure 6 et Figure 7. Pour (a)

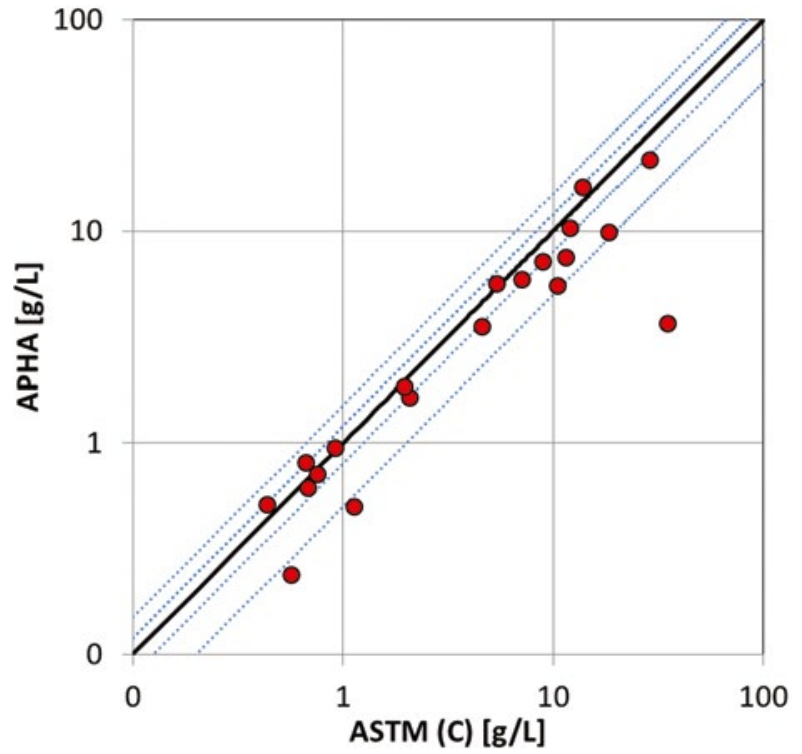

(b)

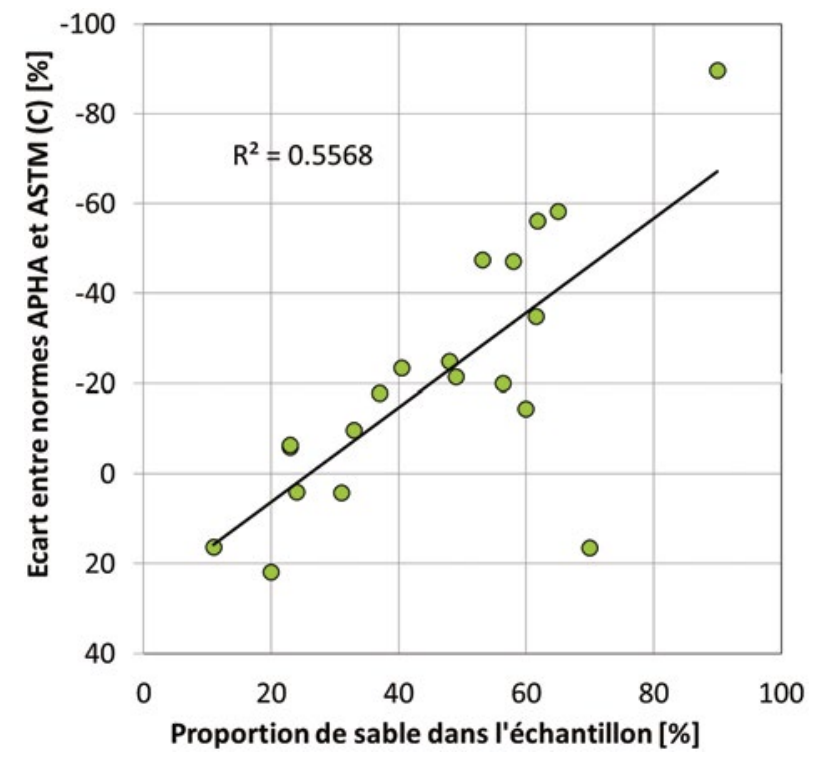

Figure 6 : a) Comparaison des concentrations ASTM (C) [2007] et APHA [2005] par filtration après sous-échantillonnage par agitation magnétique et pipettage. Les traits pointillés bleus sont les enveloppes à \pm 20 et $\pm 50 \%$, b) Ecart calculé en pourcentage entre les concentrations mesurées par les méthodes ASTM (C) [2007] et APHA [2005] en fonction du pourcentage de sable.

(a)

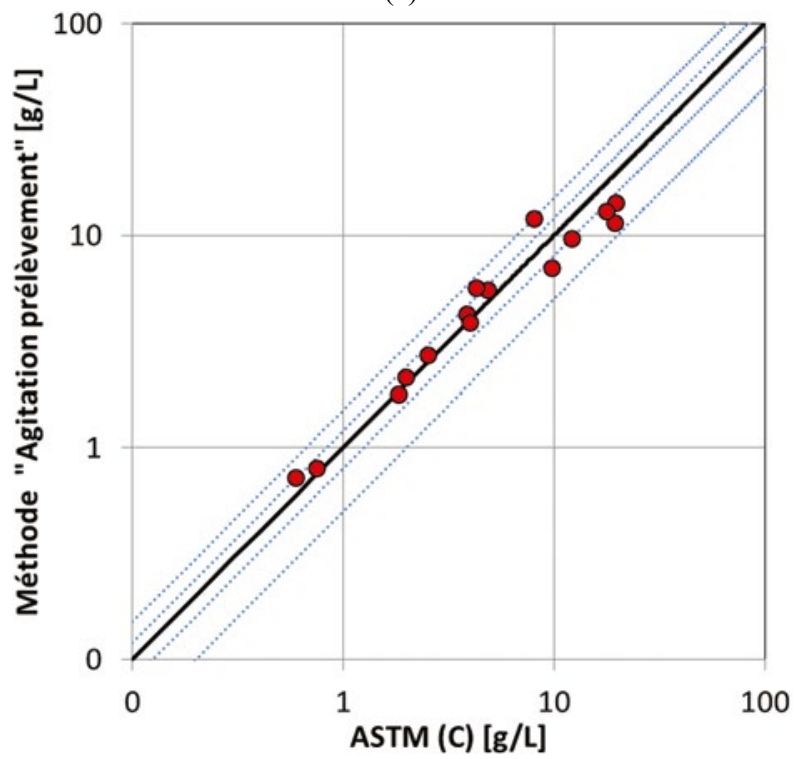

(b)

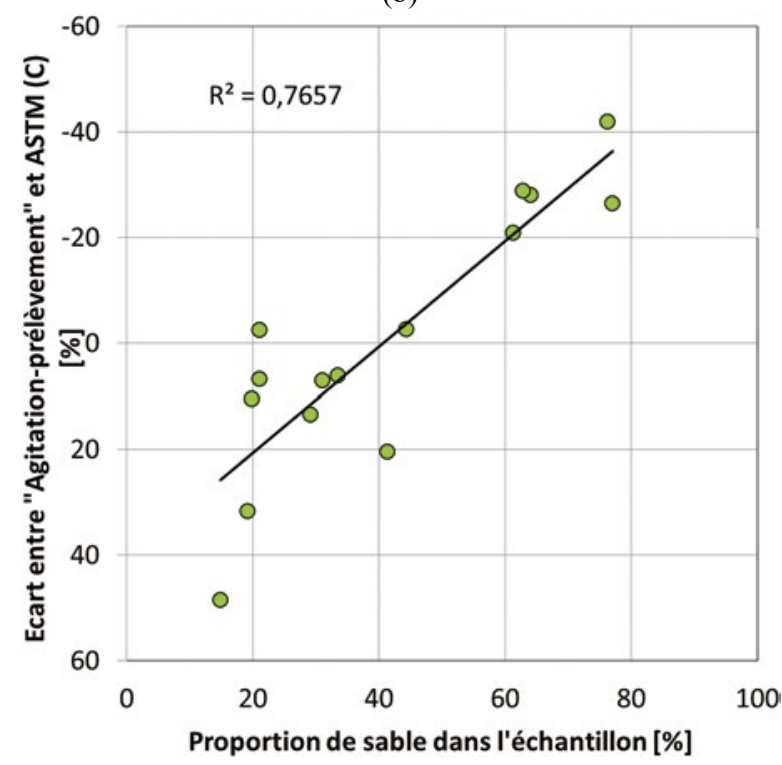

Figure 7 : a) Comparaison des concentrations ASTM (C) [2007] et par filtration après sous-échantillonnage par la méthode "Agitation-prélèvement». Les traits pointillés bleu sont les enveloppes à \pm 20 et $\pm 50 \%$, b) Ecart calculé en pourcentage entre les concentrations des méthodes ASTM (C) [2007] et par filtration après sous-échantillonnage par la méthode "Agitation-prélèvement » en fonction du pourcentage de sable. 
la méthode APHA [2005] avec sous échantillonnage par pipettage le comportement est assez similaire aux observations du premier essai avec la norme NF EN 872 [AFNOR, 2005]. On remarque une sous-estimation des concentrations quasi-proportionnelle à la quantité de particules grossières présentes dans l'échantillon, cinq échantillons ne suivent pas cette tendance, dont trois de concentration inférieure au $\mathrm{g} / \mathrm{L}$ et peu concentrés en sable. Concernant la méthode « Agitation-prélèvement », il apparaît un biais par rapport à la méthode ASTM [2007] avec une sous-estimation des concentrations des échantillons contenant un taux de sable inférieur à $50 \%$ et une sur-estimation pour les échantillons avec un taux de sable supérieur à $50 \%$.

\section{CONCLUSIONS}

Ce travail mené sur un jeu d'échantillons de l'Arc-en-Maurienne prélevés en juin 2017 dans une gamme de concentration étendue (de 0,2 à $35 \mathrm{~g} / \mathrm{L}$ ) et une gamme de taux de sable dans l'échantillon élevée (de 10 à $90 \%$ ) confirme les études menées outre-Atlantique sur les limites du sous-échantillonnage des prélèvements de rivières en présence de particules grossières. Cette étude met en lumière plusieurs points d'intérêt pour les scientifiques et gestionnaires qui s'intéressent à la mesure de la concentration et granulométrie des MES et aux flux de sédiments dans les cours d'eau :

1. La méthode de la norme américaine ASTM D3977 [2007] a été utilisée dans cette étude comme référence car elle permet de prendre en compte la totalité des particules les plus grosses. Elle a l'inconvénient d'être plus coûteuse en temps mais est mieux adaptée à l'analyse des eaux de rivière en particulier en présence de sable.

2. La présence de sable $(>63 \mu \mathrm{m})$ dans des échantillons met en défaut la méthode de sous-échantillonnage par déversement rapide du prélèvement. Cela a des conséquences directes (de $10 \%$ à $60 \%$ d'erreur) sur les résultats d'analyse en concentration mais aussi sur d'autres mesures. Par exemple si ce type de sous-échantillonnage est réalisé pour effectuer une granulométrie, on sous-estimera la présence des particules les plus grosses, restées au fond du flacon. Les variantes de méthodes de sous-échantillonnage par pipettage, agitation magnétique et méthode "Agitation-prélèvement 》 ne réduisent pas significativement les erreurs, qui restent importantes et très variables. Ces méthodes peuvent même entraîner une sur-représentation du sable et donc une surestimation des concentrations faibles si la majorité des quelques grains présents sont capturés dans le sous-échantillon.

3 . Concernant la métrologie et la normalisation : auprès des laboratoires contactés en France, on trouve une variété intéressante de méthodes de sous-échantillonnage et d'analyses pour déterminer la concentration en MES. Le seul fait que certains laboratoires, par choix méthodologique ne suivent pas la norme NF EN 872 [2005] permet d'émettre des doutes sur son domaine d'application. Le constat sur ces pratiques pose la question de la cohérence des données entre elles. Comment arbitrer entre une limite réglementaire et une mesure issue d'une analyse non normalisée, ou normalisée mais très incertaine?

Ce travail sur les échantillons de l'Arc-en-Maurienne fortement chargés en sable est généralisable à d'autres cours d'eau et fleuves, notamment en crue. En effet, d'autres jeux d'échantillons du Rhône ont été prélevés dans le cadre de l'Observatoire des Sédiments du Rhône (OSR) à Port Saint Louis, aux Rippes et à l'aval du barrage de Génissiat, dont l'analyse confirme nos présentes conclusions. Il est sans doute nécessaire en France et en Europe de lancer une réflexion sur le sujet de la mesure de la concentration en MES en présence de sable, pour élaborer ou choisir collectivement la ou les méthodes les mieux adaptées à nos besoins et nos sites d'études. Ceci pour pouvoir comparer les études menées à partir de mesure de concentration en MES : bilans de flux, courbes de calibration de turbidimètres, concentrations en polluants associés aux MES et autres modélisations hydro-sédimentaires.

\section{REMERCIEMENTS}

Nous remercions EDF-CIH pour le financement des expériences de terrain lors de la chasse de l'Arc de juin 2017. Ce travail a été réalisé dans le cadre du projet Observatoire des sédiments Rhône, financé par le FEDER (Union Européenne) et le Plan Rhône. Merci également à l'équipe métrologie d'Irstea pour la qualité de son travail et a Stéphanie Astrid Moore, qui, il y a quelques années, nous avait parlé de ce problème de sous-échantillonnage, mais nous l'avions alors sous-évalué.

\section{RÉFÉRENCES}

AfNor (2005) - NF EN 872. Qualité de l'eau - Dosage des matières en suspension - Méthode par filtration sur filtre en fibres de verre, 16.

AfNor (1997) - NF T90-105. Qualité de l'eau - Dosage des matières en suspension - Méthode par centrifugation, 10.

ApHA, American Water Works Association and the Water Environment Federation Washington D.C. (2005) - Standard methods for the examination of water and wastewater, 19 th Ed., 541.

Armijos E., Crave A., Espinoza R., Fraizy P., Santos A., Sampaio F., De Oliveira E., Santini W., Martinez Ju., Autin P., Pantoja N., Oliveira M., Filizola N. (2017) - Measuring and modeling vertical gradients in suspended sediments in the Solimões/ Amazon River. Hydrological Processes, 31 : 654-667.

Astm West Conshohocken, PA (2007) - Standard test method for determining sediment concentration in water samples. D3977-97R07, 6 .

Camenen B., Herrero A., Dramais G., Thollet F., Le Bescond C., Perret E., \& Berni C. (2015) - Field experiment on the dynamics of fine sediments over a gravel bar in an alpine gravel-bed river. Proc. RCEM conference, Iquitos, Pérou, Sept. 2015, 4.

Camenen B., Perret E., Herrero A., Berni C., Thollet F., Buffet A., Dramais G., Le Bescond C., \& Lagouy M. (2016) - Estimation of the volume of a fine sediment deposit over a gravel bar during a flushing event. Proc. River Flow conference, St Louis, Missouri, USA, July 2016, CDRom, 533-540.

CAPEl P. D., LaRson S. J. (1996) - Evaluation of selected information on splitting devices for water samples. U.S. Geological Survey Water-Ressources Investigations Report 95-4141, Sacramento, $C A$.

Cemagref (1981) - Mesure rapide des M.E.S.- Méthode dite de la " Crêpe ». Rapport Groupement d'Antony, 33.

Clark S. E., SiU C. S. (2008) - Measuring solids concentration in stormwater runoff: Comparison of analytical methods. Environmental Science \& Technology, 42(2).

Clark S. E., PitT R. (2011) - Comparison of stormwater solids analytical methods for performance evaluation of manufactured treatment devices. Journal of Environmental Engineering, 137 (11) 
DiJkman J. (1978) - Some characteristics of USP-61 and Delft Bottle suspended sediment samplers. Engineer thesis, Delft, $N L, 211$.

GIDON M. (2017) — http://www.geol-alp.com/ (consulté en juillet 2017)

Glysson G. D., Gray J. R. (2002) - Total suspended solids data for use in sediment studies. Turbidity and Other Sediment Surrogates Workshop, April 30 - May 2, 2002, Reno, NV.

Gray J. R., Glysson G. D., Torcios L. M., And Schwartz G. (2000) - Comparability of suspended-sediment concentration and total suspended solids data. U.S. Geological Survey Water-Ressources Investigations Report 00-4191, Reston, VA.

Gray J. R., Gartner J. W. (2010) - Overview of selected surrogate technologies for high-temporal resolution suspended-sediment monitoring. 2nd Joint Federal Interagency Conference, Las Vegas, NV, June 27 - July 1.

Gray J.R., And Landers M.N. (2014) - Measuring Suspended Sediment. Ahuja S. (ed.) Comprehensive Water Quality and Purification, 1 : 157-204.

Guo Q. (2006) - Correlation of total suspended solids (TSS) and suspended sediment concentration (SSC) test methods. Final Report Prepared for New Jersey Department of Environmental Protection, Division of Science, Research, and Technology, Rutgers Dept. of Civil and Environmental Engineering, Piscataway, NJ.

HuUlström F. (1935) - Studies in the morphological activity of rivers as illustrated by the river Fyris. Bulletin of the Geological Institution of the University of Uppsala, 25 : 221-527.

Horowitz A. J., Hayes T. S., Gray J. R., And Capel P. D. (1997) - Selected laboratory tests of the whole storm water sample splitting capabilities of the 14-liter churn and the Teflon cone splitters. U.S. Geological Survey Office of Stormwater Quality, Technical memorandum 97.06, Reston, VA.

Iso (2016) - 7027-1 Qualité de l'eau — Détermination de la turbidité - Partie 1: Méthodes quantitatives, 21.
Iso (2014) - 11657 Hydrometry - Suspended sediment in streams and canals - Determination of concentration by surrogate techniques, 20.

Iso (2006) - FD 6107 Qualité de l'eau - Vocabulaire - Partie 2, 39.

Iso (2005) - 4365 Liquid flow in open channels - Sediment in streams and canals - Determination of concentration, particle size distribution and relative density, 47.

Jaballah M., Camenen B., Pénard L., \& Paquier A. (2015) Alternate bar development in an alpine river following engineering works. Advances in Water Ressources, 81 : 103-113.

LARDIERE C. (2009) — Les appareils de mesure de densité en ligne. Guide d'achat : Mesures, 818, 44-51.

LEFORT P. (1991) - Transports solides dans le lit des cours d'eau dynamique fluviale. Ecole Nationale Supérieure d'Hydraulique et de mécanique de Grenoble INPG.

Niel J.F., Collilieux G., Giuliani Y. (1985) - Détermination simple et rapide des MES par la méthode du pycnomètre CNR : Application au contrôle des vidanges des retenues. Revue française des sciences de l'eau, 4 (4) : 325-340.

SElbig W.R., Bannerman R.T. (2011) - Ratios of total suspended solids to suspended sediment concentrations by particle size. Journal of Environmental Engineering, 137 (11) : 1075-1081.

Thollet F., Le Coz J., Antoine G., François P., Saguintaah L., LAUnAY M., \& CAMENEN B. (2013) - Influence de la granulométrie des particules sur la mesure par turbidimétrie des flux de matières en suspension dans les cours d'eau. La Houille Blanche, 4 : 50-56.

USEPA (1999) - Total suspended solids (TSS) EPA Method 160.2 (gravimetric, dried at $103-105^{\circ} \mathrm{C}$ ), Revised Ed., Washington, D.C., 3 .

Versini P. A., Joannis C. Et ChebBo G. (2015) — Guide technique sur le mesurage de la turbidité dans les réseaux d'assainissement. Onema, Coll. Guides et protocoles, 78. 